\title{
CHANGES IN COCAINE- AND AMPHETAMINE-REGULATED TRANSCRIPT-LIKE IMMUNOREACTIVE (CART-LI) NERVE STRUCTURES OF THE PORCINE DESCENDING COLON DURING PROLIFERATIVE ENTEROPATHY
}

\author{
SŁAWOMIR GONKOWSKI, PIOTR BURLIŃSKI, PIOTR SZWAJCA, AND JAROSŁAW CAŁKA
}

\author{
Department of Clinical Physiology, Faculty of Veterinary Medicine, \\ University of Warmia and Mazury, Olsztyn, Poland \\ slawomir.gonkowski@uwm.edu.pl
}

Received: January 9, 2012

Accepted: April 27, 2012

\begin{abstract}
The distribution pattern of CART- like immunoreactive (CART-LI) nerve structures was studied by a double immunofluorescence technique in the circular muscle layer, myenteric (MP), outer submucous (OSP), and inner submucous plexuses (ISP), as well as in the mucosal layer of porcine descending colon under physiological conditions and during proliferative enteropathy (PE). In control animals, CART-LI perikarya have been shown to constitute $3.18 \pm 0.51 \%, 3.44 \pm 0.6 \%$, and $3.33 \pm 0.72 \%$ in MP, OSP, and ISP, respectively. PE caused a decrease in the number of CART - LI neurons in MP and ISP. In OSP, the observed changes were not statistically significant. During PE, the number of CART-LI perikarya amounted to $2.18 \pm 0.27 \%, 3.07 \pm 0.06 \%$, and $0.07 \pm 0.02 \%$ within MP, OSP, and ISP, respectively. Moreover, PE caused an increase in the number of CART-LI nerve fibers in the colonic circular muscle and mucosal layers as well as in MP. This study describes for the first time changes in CART-LI nerve structures of the porcine descending colon during Lawsonia intracellularis infection, and suggests the participation of this neuropeptide in the regulation of gut functions, not only under physiological conditions, but also during pathological processes.
\end{abstract}

Key words: swine, enteric nervous system, proliferative enteropathy, cocaine- and amphetamine-regulated transcript, immunohistochemistry.

Proliferative enteropathy (PE) is a disease of porcine gastrointestinal (GI) tract caused by infection with Lawsonia intracellularis. Contrary to other inflammatory diseases of the alimentary tract, PE is characterised by proliferative changes, such as hyperplasia of immature epithelial cells in different parts of intestinal tract, most frequently within the ileum and colon (9). Moreover, it is known that infection with $L$. intracellularis induces changes in chemical coding of neurons in the enteric nervous system (ENS), both within the ileum (12) and in descending colon (4).

The aim of this study was to indicate the possible PE-induced changes in the CART-like immunoreactivity within nervous structures in the porcine descending colon. It is necessary to remark that detailed functions of CART within the GI tract have not yet been completely explained. This peptide has been found within the ENS, supplying different parts of the GI tract of numerous species $(2,5,6)$. It is known that CART may be involved in the inhibition of feeding (8), reduction of gastric acid secretion (10), and exacerbation of colonic motility (15) via regulatory circuits located within the central nervous system, but the knowledge about expression and possible functions of this peptide within ENS, especially under pathological conditions, is highly fragmentary (6). Thus, this study, which for the first time analyses the influence of PE on the CART-like immunoreactivity in porcine colonic ENS, may contribute to a better understanding possible functions of the CART peptide in pathological processes within the large intestine.

\section{Material and Methods}

The study was performed on ten immature female pigs of the Large White Polish breed (18 kg of body weight, approximately 9-week-old), which were kept under standard laboratory conditions. All surgical operations were performed in accordance with the rules approved by the Local Ethical Committee.

The pigs were divided into two groups: control group (group $\mathrm{C}, \mathrm{n}=5$ ) consisting of clinically healthy animals and experimental group (group $\mathrm{PE}, \mathrm{n}=5$ ) consisting of animals with laboratory diagnosed $L$. intracellularis infection. The diagnosis of PE was confirmed with a polymerase chain reaction (PCR) based test performed at a National Veterinary Research Institute in Pulawy, Poland. Animals were euthanised 
by an overdose of sodium thiopental (Thiopental, Sandoz, Austria; $20 \mathrm{mg} / \mathrm{kg}$ b.w. given intravenously) and then perfused transcardially with $4 \%$ buffered paraformaldehyde $(\mathrm{pH}$ 7.4) prepared ex tempore. The same parts of a descending colon (ca. $3 \mathrm{~cm}$ long, from the area where nerves from inferior mesenteric ganglia are supplying the gut) were collected from all animals, post-fixed by immersion in the same fixative for several hours and, finally, stored in $18 \%$ sucrose. Ten micrometre thick cryostat sections were subjected to routine double-labelling immunofluorescence, as described previously by Pidsudko et al. (11), using combination of antisera raised in different species and directed towards protein gene-product 9.5 (PGP 9.5; mouse monoclonal, Biogenesis, UK, working dilution 1:2,000, used as a pan-neuronal marker) and CART (rabbit monoclonal, Phoenix Pharmaceuticals, USA, 1:16,000). Primary antisera were visualised by speciesspecific secondary antisera conjugated with FITC or biotin (all from Jackson Immunochemicals, USA, in working dilution 1:800). The latter antibodies were then visualised by a streptavidin-CY3 complex (Jackson, 1:8,000). Double-labelled perikarya were evaluated under Olympus BX51 microscope equipped with epifluorescence and appropriate filter sets, counted in each ganglionated plexus (i.e. the myenteric - MP, outer submucous - OSP, and inner submucous - ISP plexus), found in the studied section (four sections per animal; only neurons with a clearly visible nucleus were included), pooled and presented as mean \pm SEM. To prevent double counting of the same perikarya, the sections were located at least $100 \mu \mathrm{m}$ apart from each other. Negative controls used in the immunofluorescence procedure included pre-absorption of the neuropeptides with an appropriate antigen, omission, and replacement control. For a semi-quantitative evaluation of the density of intraganglionic CART-LI nerve fibers, an arbitrary scale was used, where (-) meant the absence of studied fibers and $(++++)$ depicted a very dense meshwork of fibers studied. A semiquantitative evaluation of the density of the CART-LI nerve fibers within the muscular or mucosal layers was based on a count of all the profiles immunoreactive to a given antigen per observation field $\left(0.55 \mathrm{~mm}^{2}\right)$. Nerve profiles were counted in four sections per animal (in five fields per section) and the obtained data were pooled and presented as a mean \pm SEM.

Statistical analysis was performed with the Student's $t$-test (GRAPHPAD PRISM v 2.0; GraphPad Software Inc., USA). Differences were considered statistically significant at $\mathrm{P} \leq 0.05$.

\section{Results}

During the presented investigation, CART-LI nerve structures have been observed in the MP, OSP, and ISP as well as in the circle muscular and mucosal layers of the porcine descending colon under physiological condition and during PE (Table 1).
In control animals the number of CARTpositive perikarya has amounted to about $3 \%$ of all neurons within all studied kinds of intramural plexuses (Fig. 1, Table 1). Most often one or two CART-LI perikarya have been observed in the particular plexuses (Fig. 1). Interestingly, two "kinds" of ganglia considering the distribution pattern of the CART-LI structures were observed within the ISP. In the majority of ganglia in ISP lacked any of the CART-positive cell bodies (Fig. 1: ISP I); however, there were also sporadically observed ganglia that contained a few CART-LI neurons (Fig. 1: ISP I').

Moreover, CART- like immunoreactive nerve fibers were found in all layers of the porcine descending colon under physiological conditions (Table 1, Figs 1, 2). Their character depended on the studied fragment of colonic wall. In the mucosa, MP, and ISP, the density of such fibers were rather low and they were very delicate, while within circle muscular layer and OSP processes immunopositive to CART were numerous and more visible. The $L$. intracellularis infection produced changes in the distribution of CART-like immunoreactivity within the ENS of porcine colonic wall. They were expressed by a decrease in the number of CART-LI intraganglionic neurons in MP and ISP (Table 1, Fig. 1: MP, ISP). The most significant changes in the number of cell bodies were observed in the ISP, where only single CART-LI perikarya were sporadically noted during PE (Fig. 1: ISP). In the OSP, differences between control group and animals with PE were not statistically significant (Table 1, Fig. 1: OSP). A simultaneous decrease in the density of CART-positive intraganglionic fibers was observed in OSP and ISP while in MP the number of such fibers was visibly higher (Table 1, Fig. 1). Proliferative enteropathy also caused an increase in CART-positive fibers within circular muscle and mucosal layers (Fig. 2). Moreover, in PE animals these fibers were often observed within large nerve bundles and they were thicker and longer than under physiological conditions.

\section{Discussion}

The results of this study demonstrated that CART-like immunoreactivity was present in all of the studied parts of the porcine colonic nervous system. This is in accordance with previous observations in the pig and other species, including human $(2,5-7,16)$. The presence of CART-LI nerve structures in all layers of the porcine colon suggests its biological effects within the alimentary tract, and the large number of CART-LI nerve fibers in the circular muscle layer can allude to the important role of this peptide especially in control of the gut muscle functions. However, till now the knowledge concerning roles of CART within the ENS is very limited. CART was described in rats as an inhibitor of gastric emptying and gastric acid secretion (10), as well as a reducer of colonic motility via cholinergic pathways (15). 


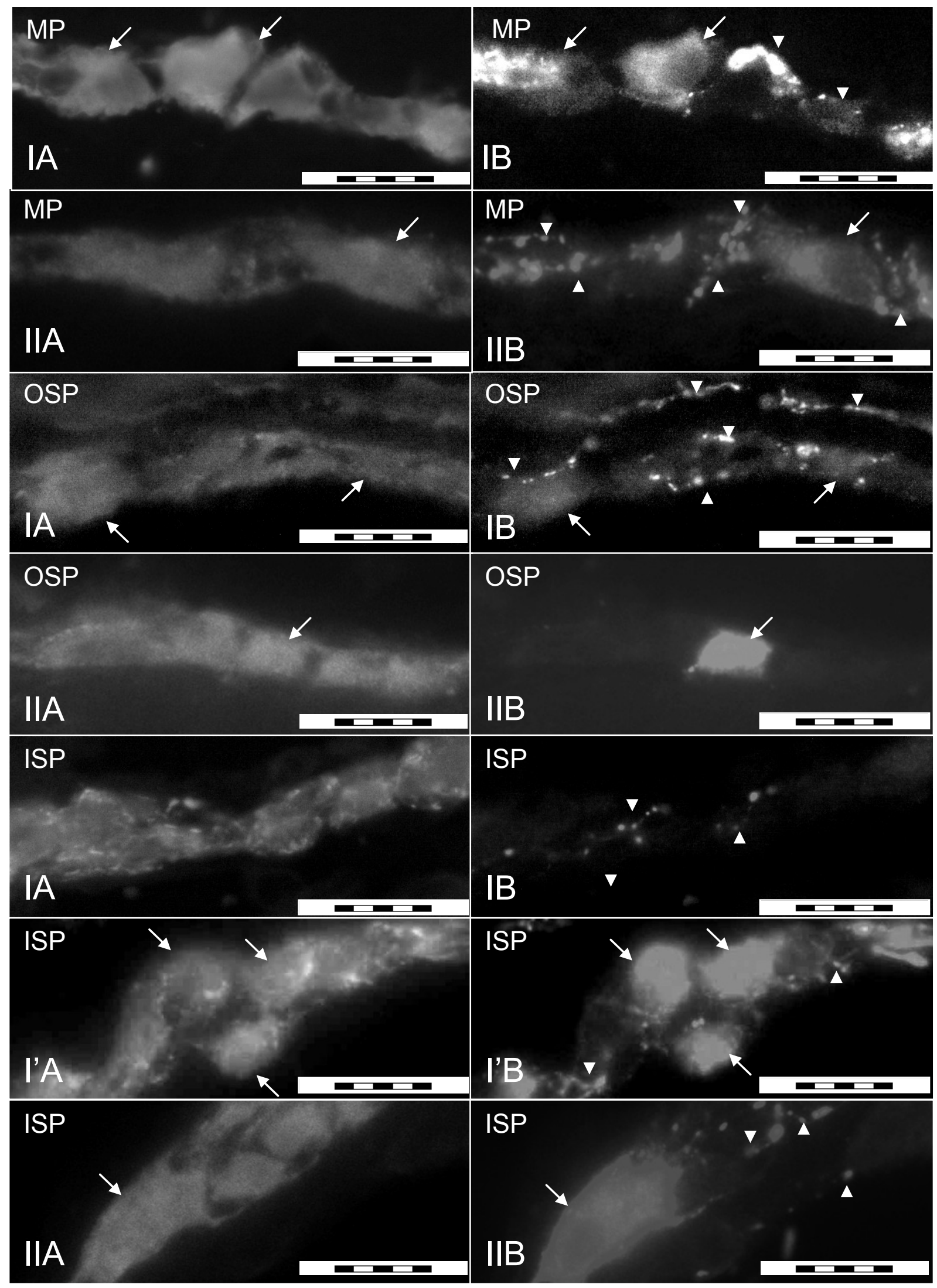

Fig. 1. MP - myenteric plexus, OSP - outer submucous plexus, and ISP - inner submucous plexus of the porcine descending colon under physiological conditions (I, I') and during proliferative enteropathy (II) immunostained for PGP 9.5 (A) and CART (B). Colocalisation of both antigens in perikarya indicated with arrows. Intraganglionic CART-LI nerve fibers indicated with arrows heads. Bar $20 \mu \mathrm{m}$. 

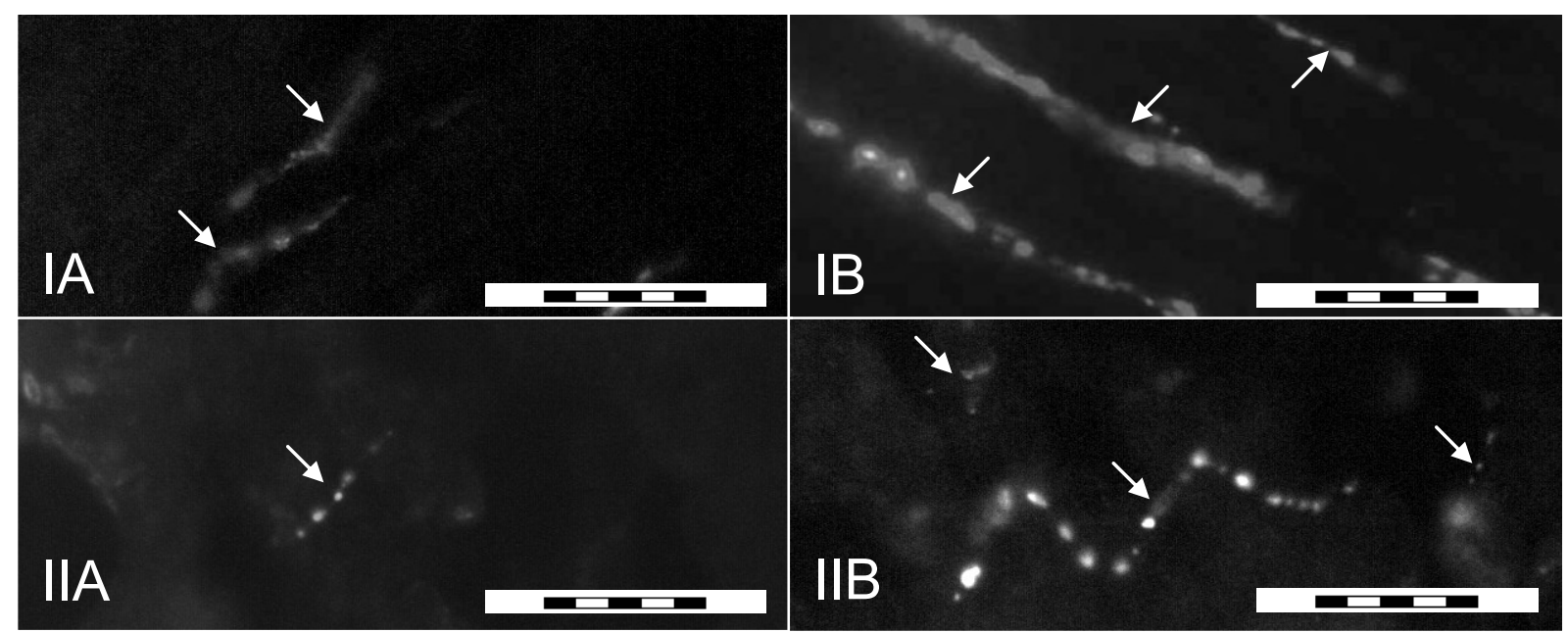

Fig. 2. Distribution pattern of nerve fibers (arrows) immunostained for CART within circular muscle (I) and mucosal layers (II) of porcine descending colon under physiological conditions (A) and during proliferative enteropathy (B). Bar $20 \mu \mathrm{m}$.

Table 1

CART-like immunoreactivity in various parts of enteric nervous system in the porcine descending colon under physiological conditions (group C) and during proliferative enteropathy (group PE)

\begin{tabular}{lccc}
\hline \multicolumn{1}{c}{ Bowel part } & & group C & group PE \\
& & & \\
\hline $\mathrm{CML}^{1}$ & & $24.18 \pm 0.35$ & $28.95 \pm 0.22^{*}$ \\
\hline $\mathrm{MP}$ & $\mathrm{CB}^{2}$ & $3.18 \pm 0.51$ & $2.18 \pm 0.27^{*}$ \\
\cline { 2 - 4 } & $\mathrm{NF}^{3}$ & + & ++++ \\
\hline OSP & $\mathrm{CB}^{2}$ & $3.44 \pm 0.60$ & $3.07 \pm 0.06$ \\
\cline { 2 - 4 } & $\mathrm{NF}^{3}$ & +++ & + \\
\hline ISP & $\mathrm{CB}^{2}$ & $3.33 \pm 0.72$ & $0.07 \pm 0.02^{*}$ \\
\cline { 2 - 4 } & $\mathrm{NF}^{3}$ & ++ & + \\
\hline S/ML & & $0.27 \pm 0.03$ & $2.44 \pm 0.23^{1}$ \\
\hline
\end{tabular}

CML - circular muscle layer; MP - myenteric plexus; OSP - outer submucous plexus; ISP - inner submucous plexus; $\mathrm{S} / \mathrm{ML}$ - submucosal/mucosal layer; $\mathrm{CB}$ - cell bodies; NF - nerve fibers. ${ }^{1}$ Average number of nerve profiles per area studied (mean \pm SEM). ${ }^{2}$ Relative frequency of particular neuronal subclasses is presented as percentage (mean \pm SEM) of all neurons counted within the ganglia stained for PGP $9.5 .{ }^{3}$ The density of intraganglionic nerve fibers positive for CART is presented.

$* \mathrm{P} \leq 0.05$

Moreover, the presented investigation revealed that the proliferative enteropathy can change CART-like immunoreactivity within the porcine descending colon and these changes have depended on the studied subdivision of colonic ENS. These results strongly suggest the role of CART in the regulation of gut function not only under physiological conditions but also during inflammatory processes.

So far very little is known about the functions of CART within the pathologically changed ENS. Two previous studies in this subject, concerning the human GI tract, revealed changes in the CART-like immunoreactivity of children ENS during Hirschprung's disease (7) as well as ulcerative colitis (6). These observations together with the results of the present study indicate the role of CART in the regulation of pathological mechanisms in GI tract. However, the functions of CART in intestinal pathology remain still unknown. Previous studies, which clearly show that
CART is able to promote the survival of rodent enteric neurons in vitro (2), strongly suggest the neuroprotective function of this peptide. Moreover, the co-localisation of CART with vasoactive intestinal polypeptide (VIP) or calcitonin gene related peptide (CGRP) in the same nervous structures of ENS $(3,16)$ can suggest similar functions of co-localised neuromediators. Thus, CART can be involved in the promotion of survival of ENS neurons in intestinal disorders and neuronal stress or injury like VIP (14), and/or takes part in sensory circuits as well as in the protection of enteric neurons in colitis like CGRP (13). On the other hand, generally it is accepted that the injury of neurons may result in an increase in the expression of neurotransmitters, which promote the regeneration of injured cells (1). In the presented study, however, a decrease in CART-positive neurons was observed in all types of ENS plexuses during L. intracellularis infection. This fact can suggest that the increase in CART expression in neurons under 
pathological factors is connected with intensive transport of this peptide to nerve endings, which is confirmed by an increase in the number of muscular and mucosal CART-LI nerve fibres in PE animals. Nevertheless, the observed changes can be the effect of a different than neuroprotective, but still unknown role of CART in ENS during gut pathological states.

To sum up, the results obtained in this study suggest the participation of CART in the function of the porcine colic ENS, not only under physiological conditions, but also during $L$. intracellularis infection. Previous studies, where CART has been described as a neuroprotective factor (2), indicate the same role for this peptide in the porcine colon; however, the relevance of CART in neural circuits controlling the functions of GI tract under various pathological conditions is not fully explained and requires further investigation. This knowledge in the future may initiate further studies on the possibility of application of this peptide as a new modern medicine in therapy of intestinal diseases.

\section{References}

1. Arciszewski M.B., Ekblad E.: Effects of vasoactive intestinal peptide and galanin on survival of cultured porcine myenteric neurons. Reg Pept 2005, 125, 185192.

2. Ekblad E.: CART in the enteric nervous system. Peptides 2006, 27, 2024-2030.

3. Ellis L.M., Mawe G.M.: Distribution and chemical coding of cocaine -and amphetamine-regulated transcript peptide (CART)-immunorective neurons in the guinea pig bowel. Cell Tissue Res 2003, 312, 265-274.

4. Gonkowski S., Burliński P., Całka J.: Proliferative enteropathy (PE)-induced changes in galanin-like immunoreactivity in the enteric nervous system of the porcine distal colon. Acta Vet (Beograd) 2009, 59, 321330.

5. Gonkowski S., Burliński P., Skobowiat C., Majewski M., Arciszewski M.B., Radziszewski P., Całka J.: Distribution of cocaine- and amphetamine-regulated transcript-like immunoreactive (CART-LI) nerve structures in the porcine large intestine. Acta Vet Hung 2009, 57, 509-520.

6. Gonkowski S., Kamińska B., Burliński P., Kroll A., Całka J.: The influence of drug-resistant colitis on the number of cocaine- and amphetamine-regulated transcript peptide-like immunoreactive (CART-LI) mucosal nerve fibres of the descending colon in children. Gastroenterology Rev 2009, 4, 147-151.

7. Gunnarsdóttir A., Wierup N., Larsson L.T., Kuhar M.J., Ekblad E.: CART-peptide immunoreactivity in enteric nerves in patients with Hirschsprung's disease. Eur J Pediatr Surg 2007, 17, 184-189.

8. Kristensen P., Judge M.E., Thim L., Ribel U., Christjansen K.N., Wulff B.S., Clausen J.T., Jensen P.B., Madsen O.D., Vrang N., Larsen P.J., Hastrup S.: Hypothalamic CART is a new anorectic peptide regulated by leptin. Nature 1998, 393, 72-76.

9. Lawson G.H., Gebhart C.J.: Proliferative enteropathy. J Comp Pat 2000, 122, 77-100.

10. Okumura T., Yamada H., Motomura W., Kohgo Y. Cocaine- amphetamine-regulated transcript (CART) acts in the central nervous system to inhibit gastric acid secretion via brain corticotrophin-releasing factor system. Endocrinology 2000, 141, 2854-2860.

11. Pidsudko Z., Kaleczyc J., Majewski M., Łakomy M., Scheuermann D.W., Timmermans J.P.: Differences in the distribution and chemical coding between neurons in the inferior mesenteric ganglion supplying the colon and rectum in the pig. Cell Tissue Res 2001, 303, 147-158.

12. Pidsudko Z., Kaleczyc J., Wąsowicz K., Sienkiewicz W., Majewski M., Zając W., Łakomy M.: Distribution and chemical coding of intramural neurons in the porcine ileum during proliferative enteropathy. J Comp Path 2008, 138, 23-31.

13. Reinshagen M., Patel A., Sottili M., French S., Sternini C., Eysselein V.E.: Action of sensory neurons in an experimental rat colitis model of injury and repair. Am J Physiol 1996, 270, G79-G86.

14. Sandgren K., Lin Z., Svenningsen A.F., Ekblad E.: Vasoactive intestinal peptide and nitric oxide promote survival of adult rat myenteric neurons in culture. $\mathrm{J}$ Neurosci Res 2003, 72, 595-602.

15. Tebbe J.J., Ortmann E., Schumacher K. Mönnikes H., Kobelt P., Arnold R., Schäfer M.K.H.: Cocaine- and amphetamine-regulated transcript stimulates colonic motility via central CRF receptor activation and peripheral cholinergic pathways in fed conscious rats. Neurogastroenterol Motil 2004, 16, 489-496.

16. Wierup N., Gunnarsdóttir A., Ekblad E., Sundler F.: Characterisation of CART-containing neurons and cells in the porcine pancreas, gastro-intestinal tract, adrenal and thyroid glands. BMC Neurosci 2007, 8, 51 DOI 10.1186/1471-2202-8-51. 